\title{
Oxygenation of the Heme-Complex Bound to Polymeric 2-Methylimidazoles
}

\author{
Hiroyuki NiSHIDE, Mikiya SEKINE, and Eishun TsuchIDA \\ Department of Polymer Chemistry, Waseda University, \\ Shinjuku, Tokyo 160, Japan
}

(Received March 10, 1982)

\begin{abstract}
Oxygenation of the heme complex of poly(1-vinyl-2-methylimidazole) (PMI) was studied in organic solvents containing $10 \mathrm{vol} \%$ water at $-30^{\circ} \mathrm{C}$. The life-time of the oxygen adduct was prolonged in $10 \mathrm{vol} \%$ water $/ N, N$-dimethylacetamide and $N$-methylpyrrolidone but oxygenation was not observed in the following $10 \mathrm{vol} \%$ water/alcohols: ethanol, butanol, and ethylene glycol. This shows a correlation to the proton accepting ability of organic solvents. The oxygen adduct was formed even in an aqueous medium for the heme complexes bound to poly(1-vinyl-2methylimidazole-co- $N, N$-dimethylacrylamide) and poly(1-vinyl-2-methylimidazole-co-1-vinylpyrrolidone) at $-30^{\circ} \mathrm{C}$ but not for heme complexes bound to the ionic copolymers: poly(1-vinyl2-methylimidazole-co-methacrylic acid) and poly(1-vinyl-2-methylimidazole-co-1-vinyl-2-methyl-3-benzylimidazolium chloride).

KEY WORDS Poly(1-vinyl-2-methylimidazole) / Iron(II) Protoporphyrin

IX / Polymer Complex / Oxygenation / Polymer Effect /
\end{abstract}

Recently, much research has been directed toward the synthesis of an iron-porphyrin complex which binds molecular oxygen reversibly as in the case of hemoglobin. ${ }^{1-4}$ The major approach has been the steric modification of porphyrin, such as picket fence iron-porphyrin, ${ }^{2}$ and has been successful in oxygen-binding in aprotic solvents or solid state. But in aqueous solutions, all iron-porphyrin derivatives were irreversibly oxidized.

The authors recently found that the heme (iron(II) protoporphyrin IX) complex of poly(1vinyl-2-methylimidazole) (PMI, Scheme 1) forms an oxygen adduct even in an aqueous solution at $-30^{\circ} \mathrm{C}{ }^{5}$ The reasons for the oxygen-binding in aqueous solution are considered to be as follows: (i) PMI forms the five-coordinate heme complex whose sixth coordination site is vacant to bind molecular oxygen (eq 1), with an unusual large formation

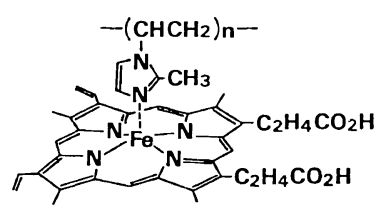

Scheme 1. constant. $^{6}$ (ii) PMI disperses and dilutes the heme complex on the polymer chain to suppress the irreversible oxidation via $\mu$-dioxo dimer (eq 2). (iii) The heme-oxygen adduct is surrounded by the hydrophobic environment of the polymer, causing the proton-driven oxidation (eq 3 ) to be retarded.

$$
\begin{aligned}
& \text { Oxygenation: } \\
& \mathrm{LFe}(\mathrm{II}) \mathrm{P}+\mathrm{O}_{2} \rightleftharpoons \mathrm{LFeP}-\mathrm{O}_{2} \\
& \text { Oxidation: } \\
& \mathrm{LFeP}-\mathrm{O}_{2}+\mathrm{LFe}(\mathrm{II}) \mathrm{P} \longrightarrow \mathrm{LFeP}-\mathrm{O}_{2}-\mathrm{LFeP} \\
& \longrightarrow \mathrm{Fe}(\mathrm{III}) \mathrm{P}-\mathrm{O}^{2-}-\mathrm{Fe}(\mathrm{III}) \mathrm{P} \\
& \mathrm{LFeP}-\mathrm{O}_{2}+\mathrm{H}^{+} \longrightarrow \mathrm{LFe}(\mathrm{III}) \mathrm{P}+\mathrm{HO}_{2}^{*}
\end{aligned}
$$

$\mathrm{Fe}(\mathrm{II}) \mathrm{P}$ and $\mathrm{Fe}(\mathrm{III}) \mathrm{P}$ : iron(II)= and iron(III) protoporphyrin IX

L: an axial ligand such as an imidazole derivative

In this paper, the solvent effect on the oxygenation of the PMI-heme complex is described first. Oxygenation was studied in $10 \mathrm{vol} \%$ water/ organic solvents for the following reasons. (i) The preparation method of heme by reducing hemin with aqueous dithionite solution is valid in these solvents. (ii) An oxygen adduct is degraded by a 
trace of water through eq 3 . On the other hand, we found that the PMI-heme complex could form a stable oxygen adduct in organic solvents containing 5-50 vol\% water, provided the temperature of these solutions was from -10 to $-30^{\circ} \mathrm{C}$ and exposed to an oxygen atmosphere. (iii) The results in a water mixed solvent are available for studying. oxygenation in aqueous media. Then heme was bound to the copolymer of 1-vinyl-2methylimidazole and the oxygenation was checked in an aqueous solution cooled to $-30^{\circ} \mathrm{C}$. The environmental effect of the copolymer surrounding the heme complex was studied and compared with the effects of organic solvents.

\section{EXPERIMENTAL}

Poly(1-vinyl-2-methylimidazole) (PMI), poly(1vinyl-2-methylimidazole-co- $N, N$-dimethylacrylamide) (PID), poly(1-vinyl-2-methylimidazoleco-1-vinylpyrrolidone) (PIP), and poly(1-vinyl-2methylimidazole-co-methacrylic acid) (PIA) were obtained by radical polymerization with $2,2^{\prime}$-azobisisobutyronitrile in methanol. Poly(1-vinyl-2methylimidazole-co-1-vinyl-2-methyl-3-benzylimidazolium chloride) (PIQ) was prepared by quaternizing PMI partially with benzyl chloride. The polymers were purified by reprecipitating them twice from ethyl ether. The amount of 2-methylimidazole units of the copolymers $(x)$ and molecular weight of the polymers $\left(M_{w}\right)$ were deter- mined by elemental analysis and vapor pressure osmometry. PMI: $M_{n} \times 10^{-4}, 2.0,4.5$, and 8.1 ; PID: $x\left(M_{n} \times 10^{-4}\right), 17 \mathrm{~mol} \%(6.3), 34$ (5.5), 51 (4.6), and 80 (4.8); PIP: $x\left(M_{n} \times 10^{-4}\right), 31 \mathrm{~mol} \%$ (4.8), 41 (4.5), and 74 (5.9); PIA: $x, 44 \mathrm{~mol} \%$, PIQ: $x\left(M_{n} \times 10^{-4}\right), 60 \mathrm{~mol}^{\%} \%$ (7.3).

Hemin (iron(III) protoporphyrin IX chloride) was purified according to Fisher's method. ${ }^{7}$ Heme was prepared by reducing hemin with an aqueous solution of sodium dithionite $\left(\left[\mathrm{Na}_{2} \mathrm{~S}_{2} \mathrm{O}_{4}\right] /[\right.$ hemin $]=$ 5) under nitrogen atmosphere. Organic solvents, $N, N$-dimethylacetamide (DMA), $N$-methylpyrrolidone (NMP), $N, N$-dimethylformamide, $N$ methylformamide, ethanol, 1-butanol, dimethyl sulfoxide, and $N$-methylacetamide, were selected according to the solubilities of PMI and heme, and the freezing points of $10 \mathrm{vol} \%$ water/organic solvents. These solvents were used after distillation in the usual way.

\section{RESULTS AND DISCUSSION}

The UV and visible spectra of the PMI-heme complex in $10 \mathrm{vol} \%$ water $/ N, N$-dimethylacetamide (DMA) at $-30^{\circ} \mathrm{C}$ are shown in Figure 1. The spectrum of the deoxy heme complex $\left(\lambda_{\max }: 427\right.$, $530,559 \mathrm{~nm})$ became the spectrum $(415,545$, $577 \mathrm{~nm}$ ) by bubbling oxygen through the solution. This assignment to the oxygen adduct was made on the basis of the following reasons. (i) The latter spectrum closely resembles that of oxy-hemoglobin

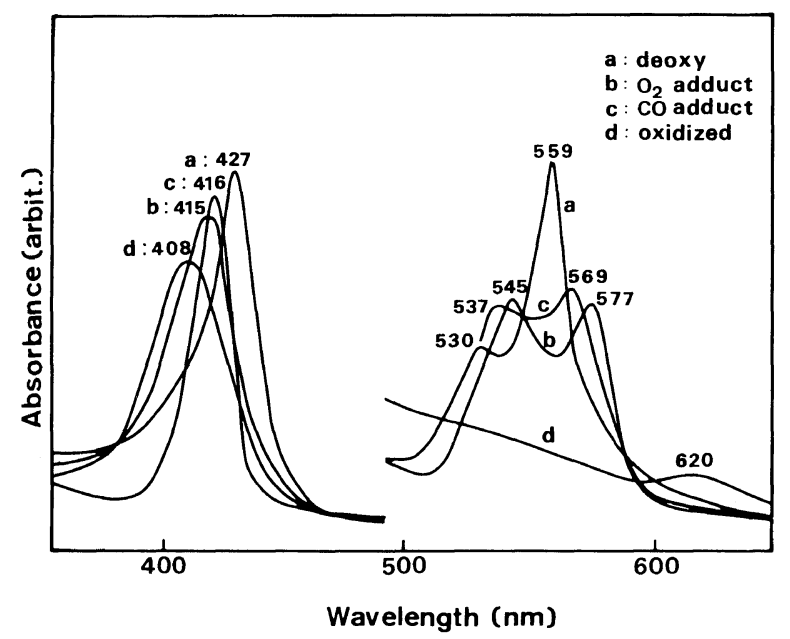

Figure 1. Ultraviolet and visible spectra of the PMI-heme complex. [heme] $=0.08 \mathrm{mM}$, [1-vinyl-2methylimidazole unit of $\mathrm{PMI}]=40 \mathrm{mM}$, in $10 \mathrm{vol} \%$ water $/ \mathrm{DMA}$ at $-30^{\circ} \mathrm{C}$. 

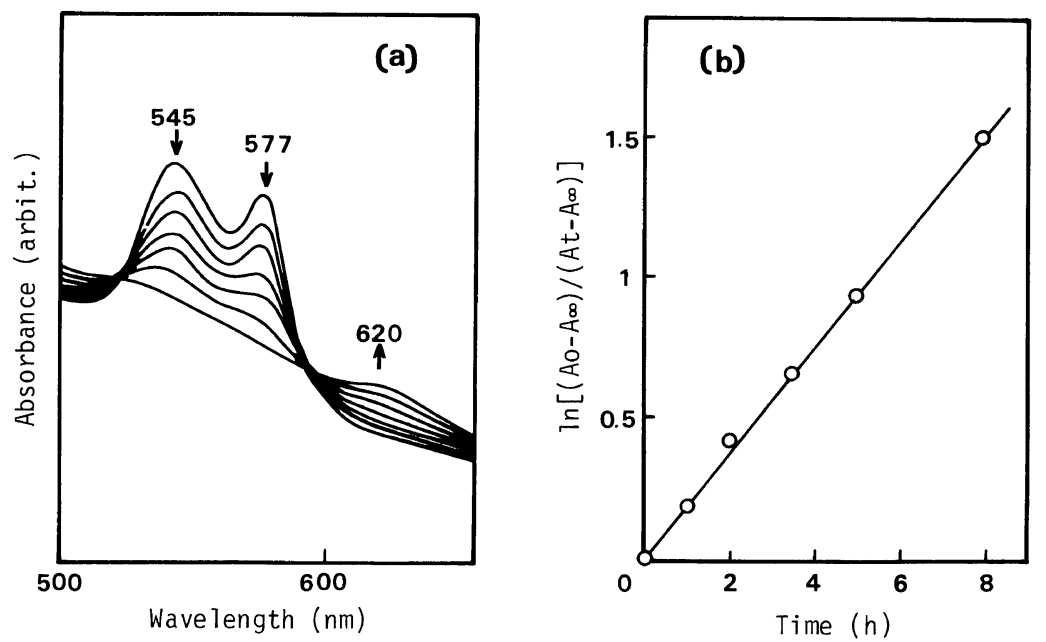

Figure 2. Spectral change of the oxygen adduct: (a), 0, 1, 2, 3.5, 5, 8, 24 $\mathrm{h}$ after exposure to air; (b), decrease in absorbance at $545 \mathrm{~nm}$. For conditions, see Figure 1.

(414, 542, $578 \mathrm{~nm}$ ). (ii) The oxidation number of the central iron (Fe(II)) did not change during oxygen exposure, since the spectrum shifted to that of the CO adduct $(416,537,569 \mathrm{~nm})$ by bubbling carbon monoxide through the oxygen adduct solution and returned to that of the deoxy complex at reduced pressure. (iii) The contribution of the reducing agents to this reversible oxygenation was ruled out by the results mentioned previously. ${ }^{5}$ It was also confirmed that the organic solvents used did not act as reducing agents of hemin under the experimental conditions. The same spectra as Figure 1 were observed in the other solvents.

When the oxygen adduct was allowed to stand under an oxygen atmosphere at $-30^{\circ} \mathrm{C}$, it gradually degraded to the PMI-hemin complex as indicated by the curve passing through the isosbestic points $(524,592 \mathrm{~nm}$, Figure 2(a)). This degradation obeyed first-order kinetics (Figure 2(b)), indicating that the irreversible oxidation of the oxygenated PMI-heme complex proceeds via a monomolecular process. Furthermore the spectrum of the oxidized product $(620 \mathrm{~nm})$ in Figure 2(a) agrees with that of the highspin PMI-hemin complex (eq 3) but not with that of the $\mu$-oxodimer of iron(III) protoporphyrin IX $(570,600 \mathrm{~nm})$ resulted from the bimolecular oxidation (eq 2).

The life-time (half-life period) of the oxygen adduct was calculated from the slope of Figure 2(b) within a $10 \%$ error. The life-time of the oxygen
Table I. Life-time of the PMI-heme oxygen adduct in $10 \mathrm{vol}^{\%} \%$ water/organic solvents at $-30^{\circ} \mathrm{C}$

\begin{tabular}{lc}
\hline \multirow{1}{*}{ Solvent } & Life-time \\
\cline { 2 - 2 } & $\mathrm{h}$ \\
\hline$N, N$-Dimethylacetamide & 3.7 \\
$N$-Methylpyrrolidone & 2.5 \\
$N, N$-Dimethylformamide & 1.1 \\
$N$-Methylformamide & 0.05 \\
Ethanol & Oxidized \\
Butanol & Oxidized \\
Ethylene glycol & Oxidized \\
Dimethyl sulfoxide & 0.5 \\
$N$-Methylacetamide & 0.2 \\
\hline
\end{tabular}

a $50 \mathrm{vol} \%$ water/organic solvent was used, because the corresponding $10 \mathrm{vol} \%$ water/organic solvent froze at $-30^{\circ} \mathrm{C}$.

[heme $]=0.08 \mathrm{mM},[1$-vinyl-2-methylimidazole unit of $\mathrm{PMI}]=40 \mathrm{mM}$.

adduct of PMI-heme at $-20^{\circ} \mathrm{C}$ was 35,32 and 34 minutes for PMIs with molecular weights of 2.0 , 4.5 , and $8.1 \times 10^{4}$, respectively. Oxygenation of heme complexes in the cooled organic solvents and cooled aqueous $\mathrm{N}, \mathrm{N}$-dimethylformamide and dimethyl sulfoxide solution has been already reported, but these oxygen adducts were not very stable. ${ }^{8-10}$ E.g., the 2-methylimidazole (2MI)-heme complex 
gave an oxygen adduct whose life-time was less than a few minutes at $-20^{\circ} \mathrm{C}$, although the spectra of the deoxy complex and oxygen and $\mathrm{CO}$ adducts of 2MI-heme $\left([2 \mathrm{MI}] /[\right.$ heme $]=10^{3}$ to $\left.10^{4}\right)$ are the same as those of PMI-heme. The PMI-ligand prolonged the life-time of the oxygen adduct.

The solvent effect on the life-time of the oxygen adduct of PMI-heme in $10 \mathrm{vol} \%$ water/organic

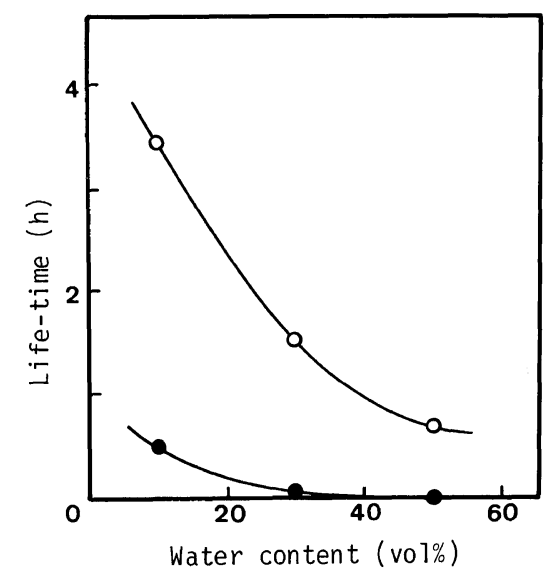

Figure 3. Effect of water concentrations on the lifetime of the oxygen adduct in the water/DMA solvent; O, PMI-heme; O, 2-methylimidazole-heme; [heme] = $0.08 \mathrm{mM}$; [1-vinyl-2-methylimidazole unit of $\mathrm{PMI}]=40$ $\mathrm{mM} ;[2 \mathrm{MI}]=400 \mathrm{mM}$; at $-30^{\circ} \mathrm{C}$. The life-time of oxygen adduct of PMI-heme in absolute DMA exceeded $5 \mathrm{~h}$, depending on the dehydration procedure. solvents at $-30^{\circ} \mathrm{C}$ is summarized in Table I. The oxygen adduct was stable in the solvents of DMA, $\mathrm{N}$-methylpyrrolidone (NMP), and $\mathrm{N}, \mathrm{N}$-dimethylformamide and relatively stable in $50 \mathrm{vol} \%$ water/ dimethyl sulfoxide (DMSO). Oxygenation was not observed in solvents such as ethanol, butanol or ethylene glycol. This life-time order does not agree with the order of the viscosities and the freezing points of the mixed solvents but is correlated to the proton accepting ability, or basicity, of the organic solvent (DMA, NMP $\gg N, N$-dimethylformamide $>$ DMSO $>N$-methylformamide). ${ }^{11}$ The oxidation process for the PMI-heme complex under this condition is considered to be the monomolecular process as mentioned above. An organic solvent of considerable proton accepting ability is considered to decrease proton activity and suppress the proton-driven oxidation process (eq 3 ).

The life-time of the oxygen adduct decreased with the water concentration in the DMA solvent, as shown in Figure 3. This is evidence that the degradation of the oxygen adduct of PMI-heme is caused by either a proton or water.

In order to obtain an oxygen adduct in aqueous media, the heme complexes bound to the copolymers of 1-vinyl-2-methylimidazole, PID, PIP, PIA, and PIQ, were prepared. PID and PIP contain the DMA and NMP moieties respectively in the polymer chains: these are the best solvents for stable oxygenation. Oxygenation was measured in the $\mathrm{pH}$

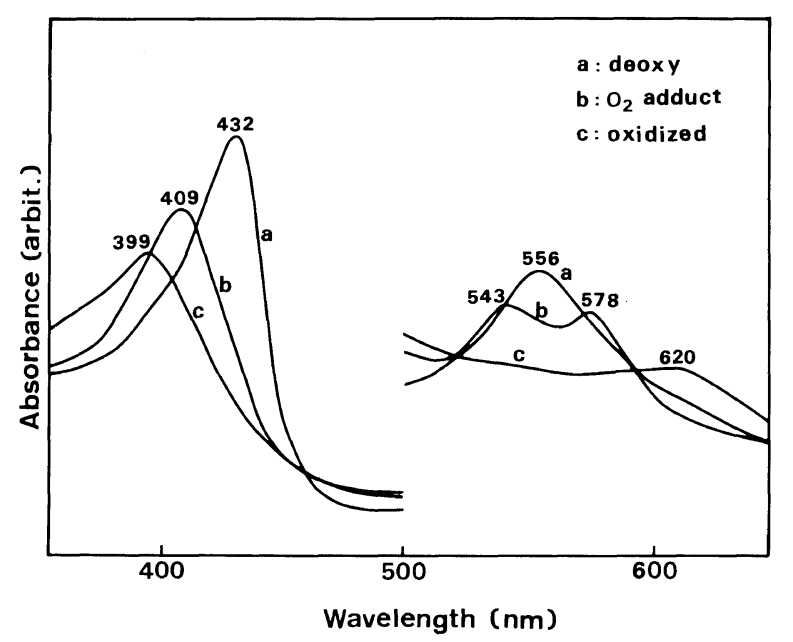

Figure 4. Ultraviolet and visible spectra of the PID-heme complex. [heme] $=0.08 \mathrm{mM}$, [1-vinyl-2methylimidazole unit of PID] $=40 \mathrm{mM}$, in $50 \mathrm{vol} \% \mathrm{pH} 10$ buffer solution/ethylene glycol at $-30^{\circ} \mathrm{C}$. 


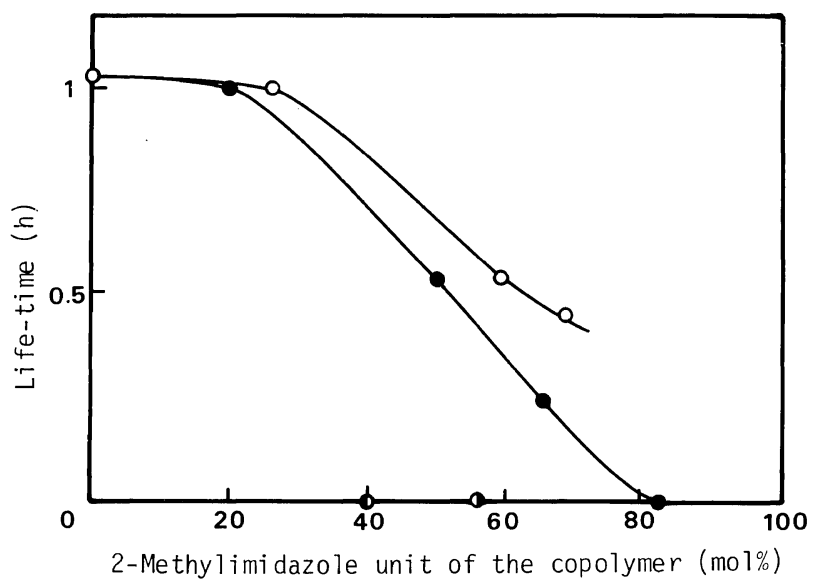

Figure 5. Oxygen adduct life-times of heme complexes bound to the copolymers: - , PID-heme; $\bigcirc$, PIPheme; P. PIA-heme; D, PIQ-heme; [heme] =0.08 mM; [1-vinyl-2-methylimidazole unit of the copolymer] $=40 \mathrm{mM}$; in $50 \mathrm{vol} \% \mathrm{pH} 10$ buffer solution/ethylene glycol at $-30^{\circ} \mathrm{C}$.

10 buffer solution $\left(0.2 \mathrm{M} \mathrm{Na}_{2} \mathrm{CO}_{3} / \mathrm{NaHCO}_{3}\right)$ containing ethylene glycol as anti-freezing agent.

When the PID-heme $(432,566 \mathrm{~nm})$ and the PIPheme $(434,557 \mathrm{~nm})$ complex solutions were cooled to $-30^{\circ} \mathrm{C}$ and exposed to oxygen, they showed the existence of an oxygen adduct $(409,543,578 \mathrm{~nm}$ and $412,546,578 \mathrm{~nm}$, respectively, Figure 4), although life-time decreased with the content of $\mathrm{N}, \mathrm{N}$ dimethylacrylamide or 1-vinylpyrrolidone units of PID or PIP (Figure 5). On the other hand, the 2MIheme complex and the heme complexes bound to the ionic copolymers, PIA and PIQ, were rapidly oxidized in the aqueous solution even at $-30^{\circ} \mathrm{C}$.

Acknowledgement. This work was partially supported by a Grant-in-Aid for Scientific Research No. 56209024 from the Ministry of Education, Science and Culture of Japan.

\section{REFERENCES}

1. For a review: F. Basolo, B. M. Hoffman, and J. A. Ibers, Acc. Chem. Res., 8, 384 (1975).

2. For a review: J. P. Collman, Acc. Chem. Res., 10, 265 (1977).

3. For a review: E. Tsuchida, J. Macromol. Sci., A13, 545 (1979).

4. For a review: T. G. Traylor, Acc. Chem. Res., 14, 102 (1981)

5. H. Nishide, H. Ohno, and E. Tsuchida, Makromol Chem., Rapid Commun., 2, 55 (1981).

6. H. Nishide, H. Ohno, and E. Tsuchida, Kobunshi Ronbunshu, 37, 641 (1980).

7. H. Fisher, "Organic Synthesis, Collective Volume 3," E. C. Horning Ed., John Wiley \& Sons, New York, 1955, p 442.

8. G. C. Wagner and R. K. Kassner, J. Am. Chem. Soc., 96, 5593 (1974).

9. W. S. Briniger, C. K. Chang, J. Geibel, and T. G. Traylor, J. Am. Chem. Soc., 96, 5597 (1974).

10. D. L. Anderson, C. J. Weschler, and F. Basolo, J. Am. Chem. Soc., 96, 5599 (1974).

11. E. M. Arnett, E. J. Mitchell, and T. S. S. R. Murty, J. Am. Chem. Soc., 96, 3875 (1974). 